\title{
The influence of oxidation on space charge formation in gamma-irradiated low-density polyethylene
}

\author{
G. Chen ${ }^{\mathrm{a}, \mathrm{b}, *}$, L.S. Zhong ${ }^{\mathrm{a}}$, H.K. Xie ${ }^{\mathrm{a}}$, H.M. Banford ${ }^{\mathrm{c}}$, A.E. Davies ${ }^{\mathrm{b}}$ \\ a State Key Laboratory of Electrical Insulation, Xian Jiaotong University, Xian, China \\ ${ }^{\mathrm{b}}$ Department of Electronics and Computer Science, University of Southampton, Southampton, UK \\ ${ }^{\mathrm{c}}$ Scottish Universities Research and Reactor Centre, East Kilbride, Glasgow, UK
}

Received 27 May 2001; accepted 4 May 2002

\begin{abstract}
The research presented in this paper investigates the role of oxidation in the formation of space charge in gammairradiated low-density polyethylene after being electrically stressed under dc voltage. Polyethylene plaques both with and without antioxidant were irradiated up to $500 \mathrm{kGy}$ using a ${ }^{60} \mathrm{Co}$ gamma source and space charge distributions were measured using the piezoelectric induced pressure wave propagation method. It has been found that a large amount of positive charge evolved adjacent to the cathode in the sample without antioxidant and was clearly associated with oxidation of the surface. The amount of charge formed for a given applied stress increased with the dose absorbed by the material. A model has been proposed to explain the formation of space charge and its profile. The charge decay after the removal of the external applied stress is dominated by a process being controlled by the cathode interfacial stress (charge injection) rather than a conventional RC circuit model. On the other hand, space charge in a sample containing antioxidant under the same applied electric stress was negligible even in the sample exposed to $500 \mathrm{kGy}$. The main process to form space charge is via charge injection rather than charge separation in the sample without antioxidant.
\end{abstract}

(C) 2003 Elsevier Science Ltd. All rights reserved.

Keywords: Gamma irradiation; Charge decay; Low-density polyethylene; Oxidation; Piezoelectric induced pressure propagation method; Space charge formation

\section{Introduction}

Polymeric materials are widely used in the electrical power industry as a dielectric because of properties such as low dielectric loss, low electrical conductivity and high breakdown strength. However, some fundamental properties such as conduction and breakdown are strongly influenced by the presence of space charge. In addition, the environment to which the materials are exposed can affect space charge formation, e.g. where nuclear radiation is present (Sessler, 1992; Gross, 1987).

\footnotetext{
*Corresponding author. Department of Electronics and Computer Science, University of Southampton, Southampton, UK. Tel.: + 44-23-8059-5166; fax: +44-23-8059-3709.

E-mail address: gc@ecs.soton.ac.uk (G. Chen).
}

Radiation can bring about not only structural changes but also give rise to trapped charge within the material. The trapping characteristics of the material can be influenced by these radiation-induced structural alternations.

In our previous papers (Chen et al., 1991, 1998a, b; Banford and Chen, 1999), the formation of space charge in gamma-irradiated low-density polyethylene (LDPE) has been studied using different techniques. It was found that the formation of space charge under de electric stress is related to several factors such as the radiation dose absorbed by the LDPE, the radiation environment, the sample thickness, the applied stress and its duration. When the irradiation is carried out in the absence of oxygen there is little charge present in LDPE compared to samples irradiated in air. On the other hand, the 
distribution of space charge is dependent on the sample thickness. In a thick sample the charge is mainly located close to the cathode surface region where the oxidation caused by irradiation occurs. In a thin sample the positive charge can extend right across the sample to the anode. All these facts suggest that oxidation caused by irradiation plays a crucial role in the formation of space charge and its distribution.

In this paper, space charge in gamma-irradiated LDPE samples with and without antioxidants has been studied under a low direct electric stress using the piezoelectric induced pressure wave propagation method (PWP) (Takada et al., 1998). Particular attention has been paid to the role of oxidation in the formation of space charge in the bulk material by comparing the amount of space charge accumulated and charge dynamics in the irradiated samples with and without antioxidant. The samples with high doses up to $500 \mathrm{kGy}$ were used in this research to enhance the effect of oxidation.

\section{The principle of PWP}

There are two major techniques widely used to measure space charge in solid dielectric materials; they are the pulsed electroacoustic (PEA) technique (Maeno et al., 1988) and the PWP technique (Laurenceau et al., 1977). Depending on how the pressure wave is generated, the latter can be further classified as the laser induced pressure pulse (LIPP) method and the piezoelectric induced pressure wave propagation method (PIPWP). The principle of the PIPWP method is shown in Fig. 1. An acoustic wave is produced by a pulsed electric stress on a piezoelectric transducer and acts as a charge probe. Any charge layer will be slightly displaced

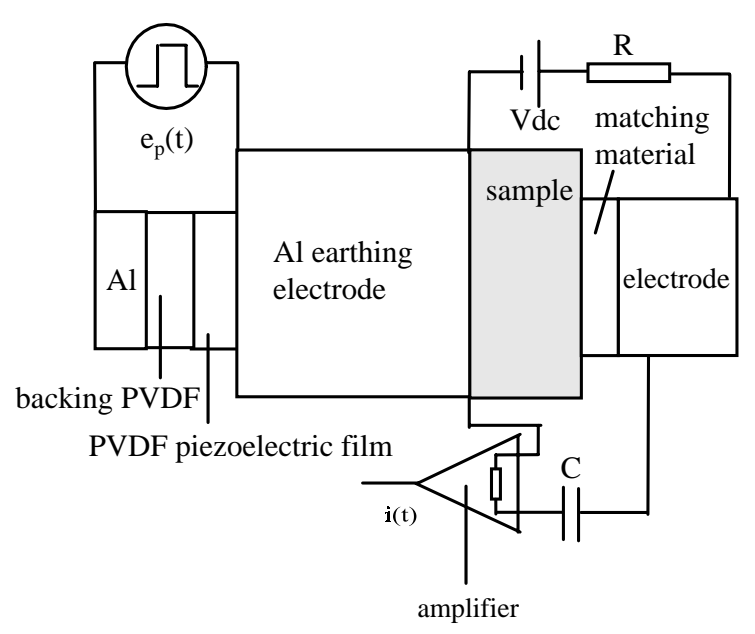

Fig. 1. Principle of the PIPWP technique. as the acoustic wave propagates through the sample. This slight displacement causes a simultaneous change in surface charge on the electrodes which will result in a current flow in the external circuit. By observing the evolution of current with time the charge profile in the material can be obtained. In order to obtain the exact charge density distribution across the sample a calibration has to be carried out at low electric stress (Takada et al., 1998). The spatial resolution of this technique depends on the width of the electric pulse, the thickness of the PVDF film and the dispersion of the material. In our present system $15 \mu \mathrm{m}$ spatial resolution has been achieved by using a $350 \mathrm{~ns}$ electric pulse and a $9 \mu \mathrm{m}$ thick PVDF film.

\section{Experimental details}

The polyethylene used in this study was HFDS-4201 supplied in granular form by Neste of Sweden. Two batches of the polyethylene were prepared, one containing no additives and the other containing $0.1 \%$ antioxidant. The exact form of this antioxidant was unknown, although it was thought to be some form of ionic compound.

Plaques of polyethylene $\sim 350 \mu \mathrm{m}$ thick were formed by a hot melt press process and were quench-cooled whilst still inside the mould. Sample plaques produced from both batches of the polyethylene, with and without the antioxidant, were characterized. High temperature permeation chromatography (HTPC) was used to determine the molecular weight distributions and differential scanning calorimetry (DSC) used to determine peak melting temperatures and lamella populations. The crystallinities of each of the polyethylene types were also calculated from measurements of the sample densities, and the microstructures of etched surfaces of the samples were observed by optical microscopy. Details of the characterization of the samples can be found elsewhere (Cartwright et al., 1996) and a summary is shown in Table 1.

Two batches of samples were irradiated in air at room temperature using a ${ }^{60} \mathrm{Co}$ gamma source. They were

Table 1

Characteristics of LDPE and LDPE with antioxidants

\begin{tabular}{lcc}
\hline Characteristic & LDPE & $\begin{array}{l}\text { LDPE with } \\
\text { antioxidant }\end{array}$ \\
\hline$M_{\mathrm{n}}$ & 9782 & 11141 \\
$M_{\mathrm{w}}$ & 58912 & 53755 \\
Density $\left(\mathrm{kg} / \mathrm{m}^{3}\right)$ & 918.6 & 922.5 \\
Crystallinity $(\%)$ & 51.0 & 53.0 \\
Melting temperature $\left({ }^{\circ} \mathrm{C}\right)$ & 108.4 & 112.5 \\
\hline
\end{tabular}


irradiated with doses between 5 and $500 \mathrm{kGy}$ at a dose rate of approximately $1 \mathrm{kGy} / \mathrm{h}$. Though $500 \mathrm{kGy}$ is not a realistic dose from the material application point of view, it was chosen to show a more pronounced effect. After irradiation, the samples were stored in freezer. Aluminium electrodes were evaporated on both sides of the sample before the space charge measurements were carried out. Test samples were electrically stressed at $5 \mathrm{kV}$ for typically $60 \mathrm{~min}$ although on one occasion this time was extended to $90 \mathrm{~min}$. The charge distributions were monitored at various times with the voltage applied. At the end of the voltage application, the distribution of space charge was measured immediately after the removal of the applied stress. In order to examine the source of charge carriers in the irradiated LDPE, one sample was annealed at $90^{\circ} \mathrm{C}$ in an oven for $8 \mathrm{~h}$ before a similar measurement was carried out.

\section{Results and discussion}

\subsection{Samples without antioxidant}

Fig. 2 shows a typical space charge result for a LDPE sample irradiated to $5 \mathrm{kGy}$. It was found that positive charges gradually developed adjacent to the cathode. The amount of charge increased with the duration of the applied stress. The formation of space charge has been attributed to the separation of positive ions and electrons created by irradiation of the material. Without the external electric stress, electrons stay very close to their parent ions, so no charge can be detected as the technique employed only measures the resultant charge (Chen et al., 1998a). With an external stress applied the charge pairs are forced to separate from each other. Electrons are swept away from the system towards the anode under the influence of the electric stress. This leaves the positive ions behind closed to the cathode.

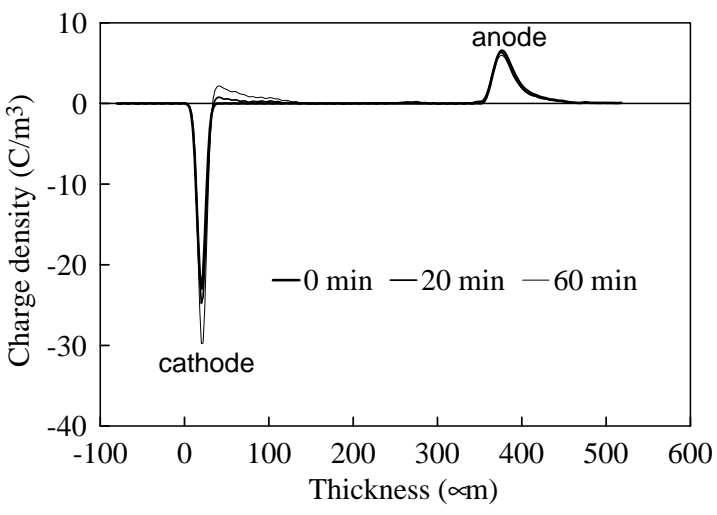

Fig. 2. Charge development in $5 \mathrm{kGy}$ sample with the field on.
At $t=0$ there is no net charge in the bulk of the sample and charges on the electrodes are due to the applied voltage. It is clear that the magnitude of the positive charge on the anode is smaller and more diffused than the charge on the cathode. In theory the amount of charge on the electrodes in this case should be the same therefore similar charge profiles should be observed. However, due to interaction with the material the pressure waves suffer the attenuation and the dispersion during propagation through the sample. Although this can be corrected through data processing (Chen et al., 1999), it is not done in the present case as the charge formed in the sample is close to the cathode where the attenuation and dispersion effects can be ignored.

Once the space charge profile $\rho(x, t)$ is obtained, the electric stress $E(x, t)$ due to space charge can be calculated based on Poisson's equation in one dimension:

$\frac{\mathrm{d} E(x, t)}{\mathrm{d} x}=\frac{\rho(x, t)}{\varepsilon_{0} \varepsilon_{\mathrm{r}}}$,

where $\varepsilon_{0}$ is the permittivity of free space and $\varepsilon_{\mathrm{r}}$ the dielectric constant of the material.

Although the dose absorbed by the material is not very high, the electric stress at the cathode is approximately $30 \%$ greater than the applied stress while the stress at the anode is reduced slightly as shown in Fig. 3. A clearer picture can be seen when the external applied stress is removed as shown in Fig. 4. In addition to the positive charge adjacent to the cathode, two small positive charge peaks are revealed close to the anode. The positive charge immediately adjacent to the anode may be caused by electron extraction at the anode. It is generally known that there are two kinds of charge carriers in terms of mobility, i.e. fast charge and slow charge (Zhang et al., 1995). Fast charges are assumed to disappear once the applied electric stress is removed. The total space charge in the bulk of a sample can be

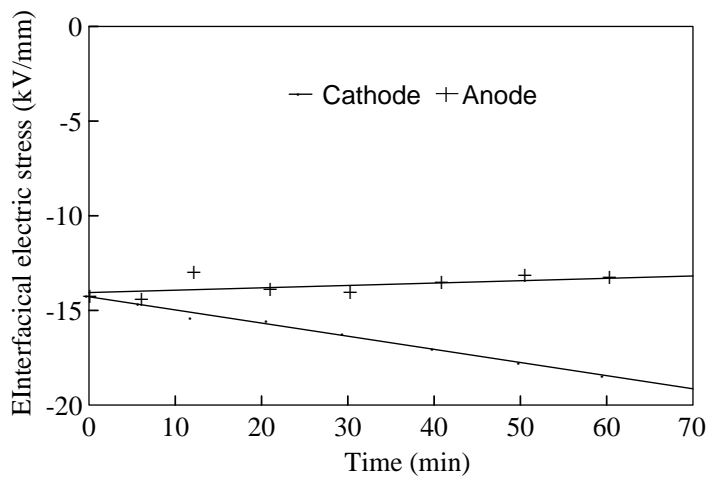

Fig. 3. Change in interfacial electric stress with time, $5 \mathrm{kGy}$ sample. 


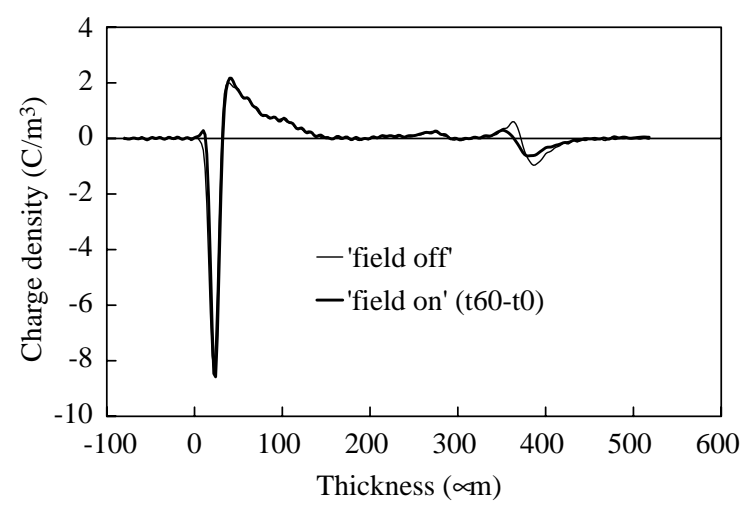

Fig. 4. Charge distribution with the field off, $5 \mathrm{kGy}$ sample.

represented as

$\rho_{\text {total }}=\rho_{\text {slow }}+\rho_{\text {fast }}$.

$\rho_{\text {total }}$ can be estimated from the difference between two measurements at a particular time and at time zero when the external voltage is applied (i.e. 'field on'). On the other hand, $\rho_{\text {slow }}$ can be obtained after the removal of the applied voltage (i.e. 'field off'). $\rho_{\text {fast }}$ can then be derived from Eq. (2). The difference between the charge profiles at $t=60$ and $t=0$ min with 'field on' shows a similar space charge distribution to that with the applied stress removed at $t=60 \mathrm{~min}$ (Fig. 4). The only difference is a small change in the positive charge adjacent to the anode. This indicates that for the present studies slow charge plays a major role and that fast charge effects are insignificant.

It is believed that the charge movement in an insulating material is closely associated with shallow traps in the sample. Chemical changes brought about through irradiation have been characterised by infrared absorption spectroscopy (Chen et al., 1998a), where it has been shown that samples irradiated at ambient temperature in air develop a strong band at $\sim 1720 \mathrm{~cm}^{-1}$. This is assigned to carbonyl groups $(\mathrm{C}=\mathrm{O})$. The infrared spectra obtained from the samples exposed to different doses show that the concentration of carbonyl groups increases with the radiation dose. Carbonyl groups resulting from the irradiation of polyethylene in air are known to alter the trapping structure and hence charge transport within the material. This group is credited with providing a shallow trap for both electrons and holes (Takai et al., 1976). It has also been reported that oxidation enhances charge injection at the cathode and extraction at the anode (Suzuoki et al., 1991). The small change in the positive peak adjacent to the anode confirms that some electron extraction has taken place.

The slow charge is caused by either deeply trapped electrons and holes or the presence of less mobile ions.

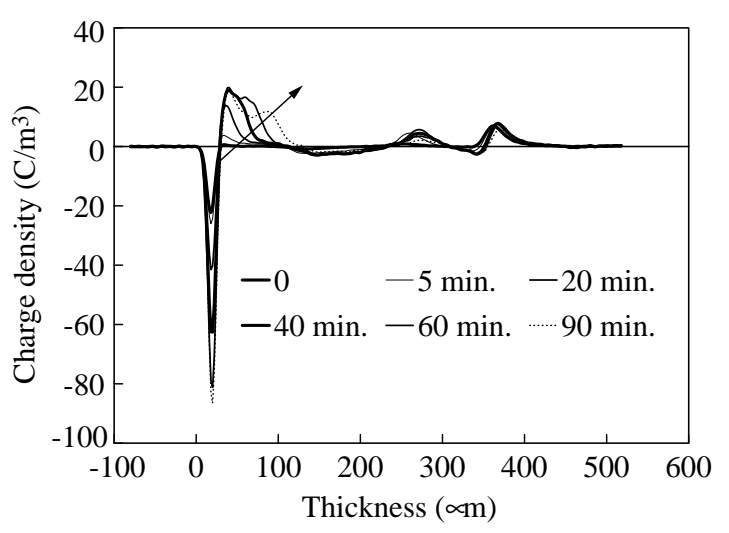

Fig. 5. Charge development with the field on, $500 \mathrm{kGy}$ sample.

In the present situation it is believed that under the influence of the applied stress the electrons have been swept away from their parent ions and entered the anode with the help of shallow traps. The less mobile ions which are left behind show the characteristics of the slow charge.

Fig. 5 depicts the development of the charge distribution over a period of $90 \mathrm{~min}$ in the sample irradiated to $500 \mathrm{kGy}$. From our previous work (Chen et al., 1998b) it has been noted that the formation of a considerable amount of charge can only occur in an oxidised region. The difference between the low and high dose samples was that for the high dose negative charge appears to accumulate in the middle of the sample followed with a small amount of positive charge. Immediately adjacent to the anode, negative charge appears rather than positive charge as in the sample with a low dose. It is also noticed that the positive peak in the middle of the samples both with high and low dose are at the same position. This has been attributed to the shallow-deepshallow trap distribution in gamma-irradiated LDPE (Chen et al., 1998a).

From Fig. 5, the charge development is quite clear. Initially, the amount of positive charge adjacent to the cathode increased with time in terms of both magnitude and volume. After $40 \mathrm{~min}$ the magnitude more or less remained the same and then decreased with time. The volume on the other hand continued to extend toward the centre of the sample. The edge of the positive peak may roughly indicate the rough boundary of the oxidised region. This agrees with the position of the second positive peak at the anode.

By integrating the charge profile, the net charge within the sample and the positive charge adjacent to the cathode can be estimated. On this basis the development of charge within the sample with time is shown in Fig. 6. The net charge within the sample is positive and shows the same trend as the positive charge adjacent to the cathode with only a small difference between them, 


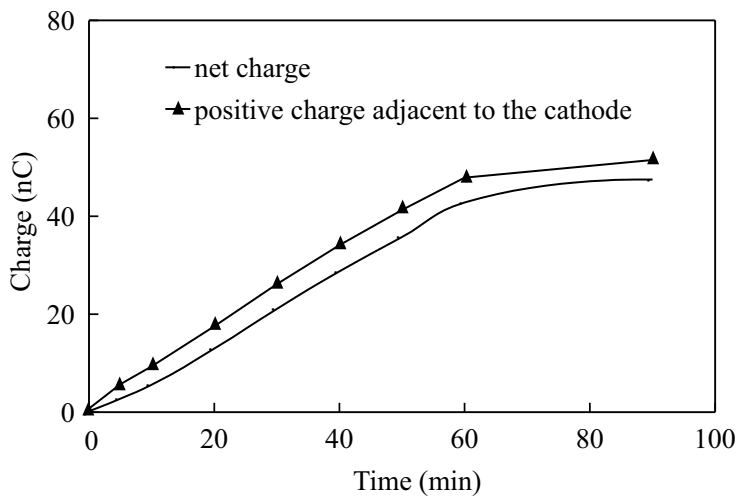

Fig. 6. Development of space charge with time, $500 \mathrm{kGy}$ sample.

indicating that the charge adjacent to the cathode is dominant. The existence of the net positive charge within the sample suggests that electron transfer is taking place at the anode. Charge accumulation increases almost linearly with time up to $60 \mathrm{~min}$ and then levels off. This agrees with the results obtained from thinner samples where a much higher electric stress was used (Chen et al., 1998b). At low electric stress, it takes a longer time to reach saturation. As previously mentioned in Chen et al. (1998b) there are two possible reasons. Firstly, all the available ion pairs produced by the gamma irradiation may have been separated. Alternatively, since the total stress at the interface (sum of the applied stress and space charge stress) is high, charge injection may take place. The charge separation rate is believed to decrease with the length of time of the applied stress. The amount of injected charge will increase with the electric stress at the cathode, but an equilibrium between charge generation and charge injection can be reached at a certain stage.

The behaviour of the charge pairs in the sample produced by gamma irradiation can be represented as in Fig. 7. Without the application of an external electric field, positive ion and electron pairs are distributed uniformly across the sample. It is postulated that electrons inside the sample tend to migrate towards the anode under the influence of the electric force. Due to changes in the trapping characteristics of the irradiated sample via oxidation at the sample surfaces, only electrons in the shallow traps (regions 1 and 3) can be detrapped. Some electrons in region 1 are captured in deep traps (region 2) on the way to the anode, while the rest of them passed through region 2 and enter region 3. The electrons in region 3 also separate from their parent ions and enter the anode. After a period of time the electric stress distribution is modified markedly by the space charge as illustrated in Fig. 7. It is expected that the amount of charge separation in a region would
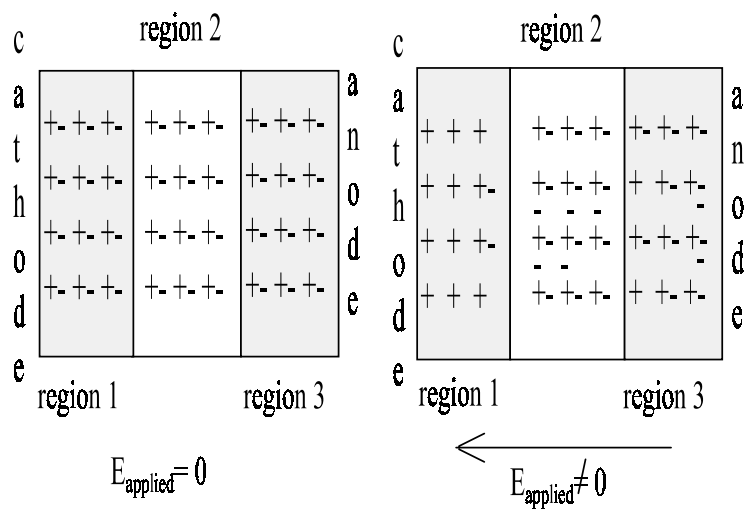

region 1 and 3 -- oxidised LDPE

Fig. 7. Schematic diagram of charge in gamma-irradiated LDPE.

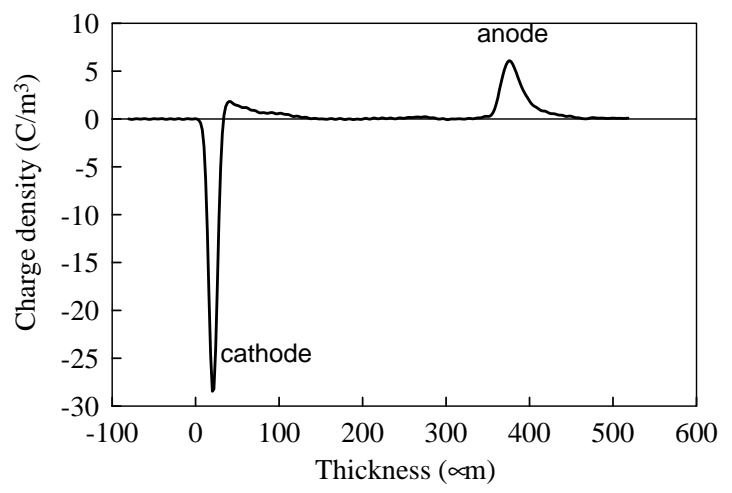

Fig. 8. Charge development in annealed $500 \mathrm{kGy}$ sample with the field on.

increase with levels of electric stress. The electric stress in region 3 is very small, which leads to the presence of a small amount of positive charge, as indicated in Fig. 5.

In order to verify the speculation outlined and the model proposed in Fig. 7, a $500 \mathrm{kGy}$ irradiated sample was placed in an oven annealing at $90^{\circ} \mathrm{C}$ for $8 \mathrm{~h}$. By doing so it was believed that thermal energy provided would release the trapped electrons and they would combine with their parent ions under the influence of Columbic force. Fig. 8 shows the charge formed in such sample after the same voltage was applied for $90 \mathrm{~min}$. It can be seen that only a small amount of positive charge is formed adjacent to the cathode. Comparing with the amount of charge formed in Fig. 5, it clearly demonstrates that annealing has a significant effect on reducing charge pairs. This suggests that majority of electrons have been recombined with their parent ions. Since there are much less charge pairs left, the amount of charge that can be separated by the applied field will be reduced. 
Based on the proposed model, numerical calculation using the hopping transportation mechanism (Lau, 2001) reveals a certain degree of similarity in charge distribution. The examination of the differences in chemical structure with depth is underway using confocal Raman microscopy and the result will be reported in the future.

The electric stress will be modified by the charge accumulated within the sample. From Fig. 5 it is expected that the main modification will take place in the interfacial stress of the cathode. The development of the stress with time is shown in Fig. 9. A linear relationship between the interfacial stress and the positive charge adjacent to the cathode can be expected as virtually all the flux lines from the charge terminate on the adjacent cathode. It can be seen that the stress enhancement caused by the positive charge adjacent to the cathode at high doses is quite significant even at a low applied stress. It is estimated that the interfacial stress in the case of the sample irradiated to $500 \mathrm{kGy}$ is over three times the applied stress.

The charge remaining within the sample after the removal of the applied stress is shown in Fig. 10. In addition, the subtraction between the time at $t=90$ and $t=0$ min with the field on is also given which shows a

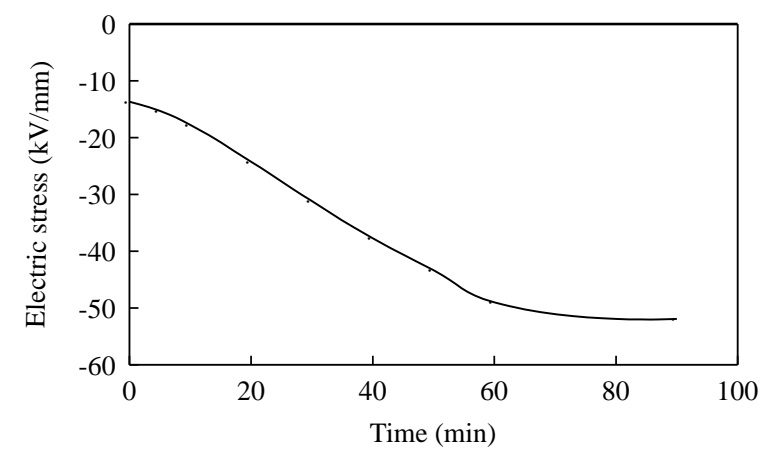

Fig. 9. Development of electric stress at the cathode with time, $500 \mathrm{kGy}$ sample.

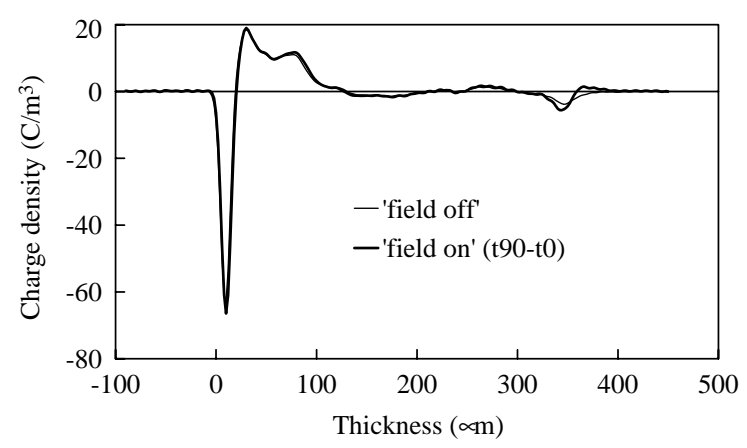

Fig. 10. Charge distribution with the field off, $500 \mathrm{kGy}$ sample. space charge distribution similar to that with the applied stress removed at $t=90 \mathrm{~min}$. A small change in the negative charge adjacent to the anode was observed, which is in agreement with the sample with a low dose.

Fig. 11 shows some typical charge profiles monitored for a period of $2 \mathrm{~h}$ after the removal of the applied stress. Generally, charge decay within the sample is governed by conduction due to electric stress, diffusion due to the concentration gradient and recombination between the positive and negative charge. In the present case, as the massive ions are the dominant charge carriers, the mobility is believed to be very small therefore diffusion can be disregarded. On the other hand, as there is a significant amount of difference between positive and negative charge within the sample, recombination between them can be considered as a second order effect compared to electrons being injected from the former cathode. By integrating, the positive charge adjacent to the cathode at different times, the relationship between charge and time can be obtained as shown in Fig. 12. An attempt has been made to fit the data into an exponential expression predicted according to the conventional RC circuit model of a dielectric. It seems that the charge

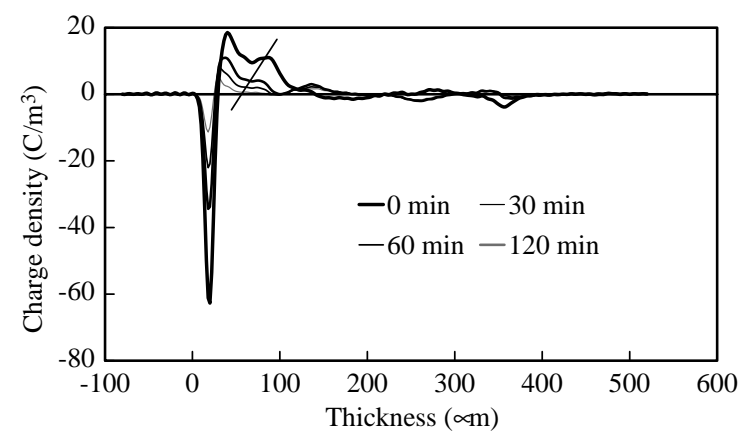

Fig. 11. Charge decay, $500 \mathrm{kGy}$ sample.

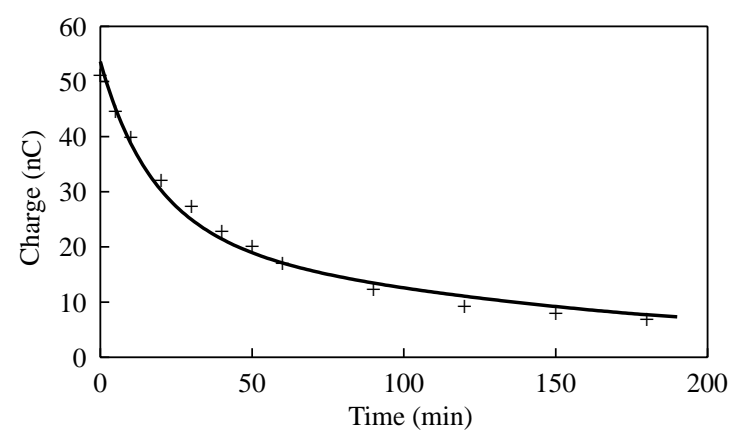

Fig. 12. Decay of positive charge adjacent to the cathode, $500 \mathrm{kGy}$ sample. 
characteristic can be represented by

$Q(t)=Q_{0} \exp \left(-\frac{t}{\tau}\right)=51.215 \exp \left(-\frac{t}{5.29}\right) \quad(\mathrm{nC})$

Here $Q_{0}$ is the initial charge at $t=0, \tau=\varepsilon \rho$ the time constant, $\varepsilon\left(=\varepsilon_{0} \varepsilon_{\mathrm{r}}\right)$ and $\rho$ are the dielectric permittivity and resistivity of the material. The value of $\varepsilon_{\mathrm{r}}$ of irradiated LDPE is measured as 2.3 at $50 \mathrm{~Hz}$ and the value of $\rho$ is around $10^{13} \Omega \mathrm{m}$ (Chen, 1990). This leads to a time constant of $\tau$ around $200 \mathrm{~s}$ which is significantly higher than the value in Eq. (3), indicating invalidity of the model.

In the present case it is believed that electron injection from the cathode is responsible for charge decay rather than conduction of positive ions themselves through the sample. Judging from what happens at the anode when the voltage is on, the electric stress at the cathode is high enough to cause electron injection. Once electrons are injected into the sample they neutralise the positive ions. Charge decay is a dynamic process mainly controlled by electric stress within the sample. The electric stress will modify the potential barrier at the interface. As the amount of charge within the sample decreases the electric stress reduces. As a consequence, the rate of charge decay reduces as can be seen in Fig. 12, where the evolution of positive charge adjacent to the cathode with time is presented. As the amount of charge within the sample decreases the electric stress reduces.

The relationship between the electric stress at the cathode interface and time can be easily obtained. If we assume that Schottky injection is responsible for the positive charge decay in the sample, then the charge decay can be examined using the numerical technique. The current density $J$ due to Schottky injection (Kasap, 1997) is given by

$J(t)=A T^{2} \exp \left(\frac{-\left(\Phi-\beta_{\mathrm{s}} E(t)^{1 / 2}\right)}{k T}\right)$,

where $A$ is the Richardson-Dushman constant, $T$ the absolute temperature, $k$ Bolzmann constant, $\Phi$ the potential barrier between aluminium electrode and the sample and $\beta_{\mathrm{s}}=\left[\mathrm{e}^{3} / 4 \pi \varepsilon_{\mathrm{r}} \varepsilon_{0}\right]^{1 / 2}$ the Schottky coefficient.

The current flow through the cathode is given by

$i(t)=J(t) S$,

where $S$ is the area of the electrode.

The amount of charge flow into the sample is therefore given by integral of $i(t)$ :

$Q_{i}(t)=\int_{0}^{t} i(t) \mathrm{d} t$.

The charge decay can be represented by

$$
\begin{aligned}
Q(t) & =Q_{0}-Q_{i}(t) \\
& =Q_{0}-A S T^{2} \int_{0}^{t} \exp \left(\frac{-\left(\Phi-\beta_{\mathrm{s}} E(t)^{1 / 2}\right)}{k T}\right) \mathrm{d} t .
\end{aligned}
$$

$Q_{0}=51.215 \mathrm{nC}$ is the initial charge obtained immediately after the removal of the applied voltage. In our experimental arrangement $S=4.762 \times 10^{-5} \mathrm{~m}^{2}$, $T=300 \mathrm{~K}$. Due to the wave nature of electrons, there is a probability that electrons may be reflected back into the metal, instead of being emitted over the potential barrier. As a consequence, the effective RichardsonDushman constant can be very low (Kasap, 1997). In the present study $A=60 \mathrm{~A} / \mathrm{m}^{2} \mathrm{~K}^{2}$ is chosen. The potential barrier $\Phi=0.8 \mathrm{eV}$ has been reported (Zebouchi et al., 1997; Fukuma et al., 1995). The calculated result based on Eq. (7) is shown in Fig. 12. Clearly there is a good agreement between the experimental and calculated results.

\subsection{Sample with antioxidant}

In the previous section, the oxidation of the sample is considered to be an important factor affecting space charge formation in additive-free LDPE. In order to confirm this, LDPE samples with antioxidants were also irradiated up to $500 \mathrm{kGy}$ and space charge was examined at the same applied stress as used above.

Fig. 13 shows the results obtained from a $500 \mathrm{kGy}$ sample. The results from the samples exposed to low doses show a similar trend but with a difference in the magnitude of the charge. Negative charge is formed adjacent to the cathode while some positive charge is formed adjacent to the anode. However, the amount of charge is very small compared to that present in the LDPE sample without antioxidants. The stress enhancement is negligible. A detailed charge profile can be seen more clearly after the removal of the applied stress as shown in Fig. 14. Oxidation is unlikely to have occurred in the sample. The results strongly support the hypothesis that oxidation during gamma irradiation is a key factor affecting space charge formation. The subtraction between the time at $t=60$ and $t=0 \mathrm{~min}$ with the field

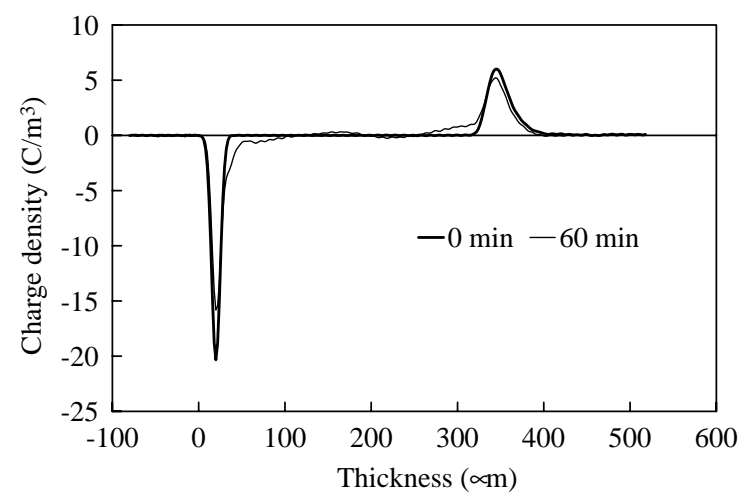

Fig. 13. Charge distribution in LDPE containing antioxidant, $500 \mathrm{kGy}$ sample. 


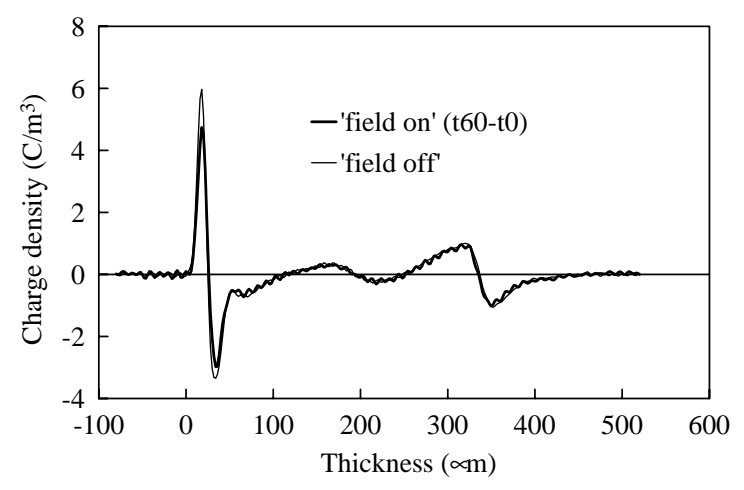

Fig. 14. Charge distribution with the field off, $500 \mathrm{kGy}$ sample.

on shows a space charge distribution similar to that with the applied stress removed at $t=60 \mathrm{~min}$, indicating the presence of slow charge. However, the modification of the surface of the sample due to irradiation may be responsible for the slightly higher charge injection under the same applied stress when exposed to higher doses.

Why the charge distribution in LDPE containing antioxidants shows this kind of profile is not clear. Homocharge is generally considered to be due to charge injection from the electrodes. As the applied stress in the present study is less than $15 \mathrm{kV} / \mathrm{mm}$, the injection rate must be very moderate. This may explain why the charge present adjacent to both the electrodes is small. Deep traps originating from the interfaces between amorphous and crystalline regions may be responsible for the slow charge. From previous work, it is known that a very small concentration of charge pairs can be separated in LDPE when deep traps are involved. Hence the positive and negative charge within the sample may be associated with the separation of the charge pairs produced by irradiation.

Unlike the sample without antioxidants where the slow charge consists of less mobile ions, in the sample containing antioxidants the slow charge can be mainly attributed to the deeply trapped electrons and holes. Under the influence of the modest electric field, a small amount of electrons can be injected from the cathode and extracted from the anode. Since there is no significant oxidative process occurring in the sample, the shallow traps associated with oxidation are not present. Therefore the electrons and holes can only be trapped by the deep traps such as interfaces between crystalline and amorphous regions.

Charge decay after the removal of the applied stress was also monitored. Compared with the sample without antioxidants, charge decay is slow. There may be two reasons for this slow process. The first is that injected charges are captured in deep traps originating from interfaces between amorphous and crystalline regions. As a result the energy obtained from thermal vibration is not high enough to release the deeply trapped charge. The second reason may be attributed to the low electric stress within the sample.

\section{Conclusions}

The evolution and behaviour of space charge in gamma-irradiated LDPE with and without antioxidant at low applied electric stresses have been studied using the PWP technique. The following conclusions may be drawn from the present study.

There are distinct differences in charge characteristics of the two types of LDPEs. Samples without antioxidant show significant positive charge in the region close to the cathode after being electrically stressed. The amount of charge increases with the absorbed radiation dose and shows a time dependence in that it increases with time initially and then saturates. The space charge results from the 'fields off' measurements indicating that slow charge dominates. A model based on surface oxidation has been proposed and the charges left behind during electric stressing are considered to be ionic charges while electrons are swept away and absorbed by the anode.

Once the applied electric field is removed, the charges accumulated in the bulk of the sample decrease with time. The charge decay cannot be simply explained by RC circuit model. Numerical simulation reveals that the decay of positive charges is due to Schottky injection of electrons from the cathode.

However, no significant charge can be found in gamma-irradiated samples containing antioxidant. Thus the formation of space charge is clearly related to the degree of oxidation. This is consistent with our earlier observation. The decay mechanism in the sample containing antioxidant is dominated by detrapping of charge carriers rather than the electrode effect.

At low applied electric stress it is possible that the space charge remained in the sample can be used to assess the ageing taken place in the material.

\section{Acknowledgements}

One of the authors (GC) thanks the Ministry of Education in China for funding his visiting position at the State Key Laboratory of Electrical Insulation.

\section{References}

Banford, H.M., Chen, G., 1999. Investigation of the distribution of space charge in gamma irradiated low-density polyethylene. Radiat. Phys. Chem. 54, 523-530.

Cartwright, G.A., Davies, A.E., Swingler, S., Vaughan, A., 1996. Effect of an antioxidant additive on morphology and 
space charge characteristics of low-density polyethylene. Proc. IEE Sci. Meas. Tech. 143, 26-34.

Chen, G., 1990. Permanent changes in electrical properties of gamma-irradiated LDPE. Ph.D. thesis, Strathclyde University, UK.

Chen, G., Fourace, R.A., Banford, H.M., Tedford, D.J., 1991. The effects of gamma-irradiation on thermally stimulated discharge current spectra in low-density polyethylene. Radiat. Phys. Chem. 37, 523-530.

Chen, G., Banford, H.M., Davies, A.E., 1998a. Space charge formation in gamma-irradiated low-density polyethylene. IEEE Trans. Dielectr. Electr. Insul. 5, 51-57.

Chen, G., Banford, H.M., Davies, A.E., 1998b. Influence of Radiation Environments on Space Charge Formation in Gamma-Irradiated Low Density Polyethylene. ISEIM, Toyohashi, Japan, pp. 113-116.

Chen, G., Davies, A.E., Vazquez, A., 1999. Space Charge Measurement in Dispersive Dielectrics. Jicables, Versailles, France, pp. 733-738.

Fukuma, M., Nagao, M., Kosaki, M., 1995. Numerical analysis on transient space charge distribution in XLPE. Fifth ICSD, Leicester, UK, pp. 139-143.

Gross, B., 1987. In: Sessler, G.M. (Ed.), Electret. Springer, Berlin, pp. 217-284.

Kasap, S.O., 1997. Principles of Electrical Engineering Materials and Devices. McGraw-Hill, New York.

Lau, S., 2001. Private communication.
Laurenceau, P., Dreyfus, G., Lewiner, J., 1977. New principle for the determination of potential distributions in dielectrics. Phys. Rev. Lett. 38, 46-49.

Maeno, T., Futami, T., Kushibe, H., Takada, T., Cooke, C.M., 1988. Measurement of spatial charge distribution in thick dielectrics using the pulsed electroacoustic method. IEEE Trans. Electr. Insul. 23, 433-439.

Sessler, G.M., 1992. Charge dynamics in irradiated polymers. IEEE Trans. Electr. Insul. 27, 961-973.

Suzuoki, Y., Furuta, H., Yamada, H., Han, S.O., Mizutani, T., Ieda, M., Yoshifuji, N., 1991. Study of space charge in polyethylene by direct probing - effects of oxidation. IEEE Trans. Electr. Insul. 26, 1073-1079.

Takada, T., Tanaka, Y., Adachi, N., Qin, X., 1998. Comparison between the PEA method and PWP method for space charge measurement in solid dielectrics. IEEE Trans. Dielectr. Electr. Insul. 5, 944-951.

Takai, Y., Mori, K., Mizutani, T., Ieda, M., 1976. Investigation of traps in g-irradiated polyethylene by photostimulated detrapping current analysis. Jpn. J. Appl. Phys. 15, 23412347.

Zebouchi, N., Se-Ondoua, M., Malec, D., Hoang, T.G., 1997. Modelling of transient space charge distribution in polymer. J. Electrostatics 40\&41, 495-501.

Zhang, Y., Lewiner, J., Alquie, C., Hampton, R.N., 1995. Space charges of different mobilities: a novel data processing method. J. Appl. Phys. 77, 5195-5200. 